\title{
Some Properties of Algebra of Quotients with Bounded Evaluation of a Norm Ideal on Complex Banach Space
}

\author{
Mohammed Th. Al-Neima \\ mohammedmth@gmail.com \\ Department of Civil Engineering \\ College of Engineering
}

University of Mosul, Mosul, Iraq

\author{
Amir A. Mohammed \\ amirabdulillah64@gmail.com \\ Department of Mathematics
}

College of Education for Pure Sciences

Accepted on: 08/03/2020

Received on: 05/01/2020

\section{ABSTRACT}

Cabrera-Mohammed proved that the imbedding of a norm ideal on Hilbert space in algebra of quotients with bounded evaluation is continuous with other properties. In this paper we improve this result by using complex Banach space instated of Hilbert space.

Keyword: algebra of quotients with bounded evaluation, bounded algebra of quotients, totally prime algebra.

$$
\begin{aligned}
& \text { بعض خصائص جبر القسومات ذات القيم المقيدة لمثالية المعيار على فضاء باناخ المعقد } \\
& \text { محمد ننون النعمة عامر عبد الاله محد }
\end{aligned}
$$

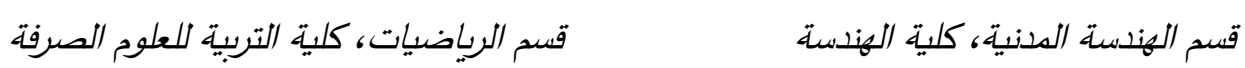

$$
\begin{aligned}
& \text { جامعة الموصل، الموصل، العراق }
\end{aligned}
$$

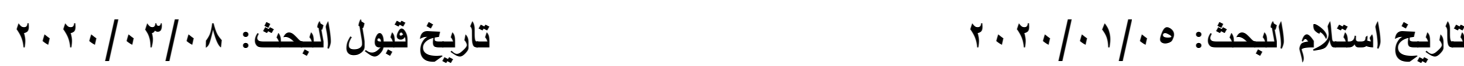

$$
\begin{aligned}
& \text { فضاء هلبرت. } \\
& \text { الكلمات المفتاحية: جبر القسومات ذات القيم المقيدة، جبر القسومات المقيدة، الجبر الأولي الكلي. }
\end{aligned}
$$

\section{Introduction}

Normalization of a subalgebra of Martindale of algebra of quotients of prime algebra was studied first in 1986 by Martin Mathieu in his thesis [8] by using ultraprime normed algebra which known as bounded algebra of quotients. In 1990, CabreraRodriguez defined totally prime algebra and they proved every ultraprime normed algebras are totally prime algebras [5, Theorem 1, p. 5]. In 2001, Cabrera-Mohammed extended the study of normalization of a subalgebra of Martindale of algebra of quotients of ultraprime normed algebra to totally prime algebra. The new subalgebra is called algebra of quotients with bounded evaluation.

In [3], Cabrera-Mohammed proved that the imbedding of a norm ideal on Hilbert space in algebra of quotients with bounded evaluation is continuous and the algebra of quotients with bounded evaluation is a right ideal of bounded algebra of quotients. 
Oure aim is to extend the work of Cabrera-Mohammed in [3, proposition 3, $\mathrm{p}$. 121] by using norm ideal on complex Banach space instated of Hilbert space. All algebras which will be used in this paper are associative algebras.

\section{Bounded algebra of quotients and algebra of quotients with bounded evaluation of prime normed algebra.}

Recall that $A$ is prime algebra, if $I J=0$ then either $I=0$ or $J=0$ for any $I, J$ ideals of $A$. Let $A$ be an algebra and $x, y \in A$ the linear operator $M_{x, y}$ is defined on $A$ such that $M_{x, y}(z)=x z y$ for all $z \in A$. A normed algebra $A$ is called ultraprime if there exists a constant $c>0$ such that $c\|x\|\|y\| \leq\left\|M_{x, y}\right\|$ for all $x, y \in A \cdot[8$, definition 1.2, p.13].

Let $A$ be a prime normed algebra, the right bounded algebra of quotients of $A$ is defined to be subalgebra of $Q^{r}(A)$ Martindale algebra of quotients of $A$. It denoted by $Q_{b}^{r}(A)$ and given by $Q_{b}^{r}(A)=\left\{q \in Q^{r}(A):\right.$ I ideal of $A, q I \subseteq A, L_{q}^{I}$ is bounded $\}$

Let $q \in Q_{b}^{r}(A)$ the norm of $Q_{b}^{r}(A)$ is given by $\|q\|_{r}=\inf \left\{\left\|L_{q}^{I}\right\|:\right.$ I ideal of $A, q I \subseteq A, L_{q}^{I}$ is bounded $\}$

Where $L_{q}^{I}$ is a linear operator from $I$ into $A$ such that $L_{q}^{I}(x)=q x$ for all $x \in I$. $Q_{b}^{r}(A)$ it well be right bounded algebra of quotients for prime algebra $A$ with identity by $\left[8\right.$, proposition 2.6, p. 21] with algebra seminorm $\|\cdot\|_{r}$ by $[8$, Lemma 2.12 , p. 26]. When $A$ is ultraprime normed algebra, then $Q_{b}^{r}(A)$ be ultraprime normed algebra too by [9, Theorem 4.1(b), p. 307]. The left bounded algebra of quotients has similar definition and denoted by $Q_{b}^{l}(A)$ with algebra seminorm denoted by $\|\cdot\|_{l}$.

The multiplication algebra $M(A)$ for algebra $A$ is a subalgebra of $L(A)$ generated by left and right multiplication operators $\left\{R_{a}, L_{a}: a \in A\right\}$ and the identity operator $I d_{A}$. Let $A$ be an algebra and $x, y \in A$ the bilinear operator $N_{x, y}$ from $M(A) \times M(A)$ into $A$ defined by $N_{x, y}(F, G)=F(x) G(y)$ for all $F, G \in M(A)$. Recall that a normed algebra $A$ is totally prime if there exists a constant $c>0$ such that $c\|x\|\|y\| \leq\left\|N_{x, y}\right\|$ for all $x, y \in A$. The multiplication algebra $M(A)$ is a subalgebra of $B L(A)$ which is normed under operator norm see [4, p. 128].

For defining algebra of quotients with bounded evaluation for prime normed algebra we need the following definition.

Let $A$ be a prime algebra and $A^{1}$ is the unitization for algebra $A$ of $Q^{r}(A)$ when $A$ without identity. Let $I$ be an ideal of $A$, the set $I^{r}$ is given by

$$
I^{r}=\left\{\sum_{i=1}^{n} M_{a_{i}, x_{i}}, n \in \mathbb{N}, a_{i} \in A^{1}, x_{i} \in I, 1 \leq i \leq n\right\}
$$

Cabrera-Mohammed in [3, lemma 1, p. 116] proved that $I^{r}$ is an ideal of $M(A)$.

Let $A$ be prime algebra, $q \in Q^{r}(A)$ and $I$ non-zero ideal of $A$ such that $q I \subseteq A$. The linear operator $E_{q}^{I^{r}}$ from $I^{r}$ into $A$ is defined by

$$
E_{q}^{I^{r}}(F)=F(q)=\sum_{i=1}^{n} M_{a_{i}, x_{i}}(q)
$$

Let $A$ be a prime normed algebra, the right algebra of quotients with bounded evaluation for $A$ is defined to be subalgebra of $Q^{r}(A)$. It denoted by $Q_{b e}^{r}(A)$ and given by 


$$
Q_{b e}^{r}(A)=\left\{q \in Q^{r}(A): \text { I ideal of } A, q I \subseteq A, E_{q}^{I^{r}} \text { is bounded }\right\}
$$

Let $q \in Q_{b e}^{r}(A)$ the norm of $Q_{b e}^{r}(A)$ is given by

$$
\|q\|_{r e}=\inf \left\{\left\|E_{q}^{I^{r}}\right\|: \text { I ideal of } A, q I \subseteq A, E_{q}^{I^{r}} \text { is bounded }\right\}
$$

$Q_{b e}^{r}(A)$ it will be right algebra of quotients with bounded evaluation for prime normed algebra $A$ with seminorm $\|\cdot\|_{r e}$ by [3, theorem 1, p. 117], when $A$ is totally prime algebra then $Q_{b e}^{r}(A)$ it well be totally prime algebra too by [3, Theorem $5, \mathrm{p}$. 129]. The left algebra of quotients with bounded evaluation has similar definition and denoted by $Q_{b e}^{l}(A)$ with algebra seminorm denoted by $\|\cdot\|_{l e}$.

\section{Properties for right algebra of quotients with bounded evaluation of a norm ideal on complex Banach space.}

Let $X, Y$ be two normed spaces on a filed $K$, the linear operator $T$ from $X$ to $Y$ is called finite rank operator if $T \in B L(X, Y)$ such that $T(X)$ is finite dimension subspace of $Y$. We denoted to set of all finite rank operators from $X$ to $Y$ by $F B L(X, Y)$ which is an subspace of $B L(X, Y)$. If $X=Y$ then $F B L(X)$ is an ideal of $B L(X)$. Let $X$ be a nonzero normed space on filed $K$ and $X^{\prime}$ is the dual space of $X$. Let $y \in X$ and $f \in X^{\prime}$ the operator $y \otimes f$ defined on $X$ such that $(y \otimes f)(x)=f(x) y[6,1.412$, p. 73].

We say that $A$ is norm ideal, if $A$ is a non-zero ideal of $B L(X)$ with norm $\|\cdot\|$ satisfy the following:

i. $\|x \otimes f\|=\|x\|\|f\|$ for all $x \in X$ and $f \in X^{\prime}$.

ii. $\|F T G\| \leq\|F\|_{\infty}\|T\|\|G\|_{\infty}$ for all $T \in A$ and $F, G \in B L(X)$

Also $\|T\|_{\infty} \leq\|T\|$ for all $T \in A$

[7, definition 6.1.141, p. 409]

The following theorem is improve to theorem for Cabrera-Mohammed in [3, Theorem 2, p.120] which is used complex Banach space instated of Hilbert space

\section{Theorem 3.1}

Let $A$ be norm ideal on complex Banach space $X$. Then

For proof see [1, Theorem 2.2].

$$
\left(Q_{b}^{r}(A),\|\cdot\|_{r}\right)=\left(B L(X),\|\cdot\|_{\infty}\right)
$$

Cabrera-Mohammed in [3, proposition 3, p. 121] proved that the imbedding of norm ideal on Hilbert space in algebra of quotients with bounded evaluation is continuous with other properties. We give the same result which have given in [3, proposition 3, p. 121] but we use the complex Banach space in our proof instead of Hilbert space.

\section{Proposition 3.2}

Let $(A,\|\cdot\|)$ be norm ideal on complex Banach space $X$ then $Q_{b e}^{r}(A)$ is a right ideal of $B L(X)$ and $\|\cdot\|_{r e}$ is norm have the following properties:
i. $\|T\|_{r e} \leq\|T\|$
ii. $\|S\|_{\infty} \leq\|S\|_{r e}$
iii. $\left\|x \otimes y^{\prime}\right\|_{r e}=\|x\|\left\|y^{\prime}\right\|$
iv. $\|S F\| \leq\|S\|_{r e}\|F\|_{\infty}$
v. $\|S G\|_{r e} \leq\|S\|_{r e}\|G\|_{\infty}$

For all $T \in A, S \in Q_{b e}^{r}(A), x \in X, y^{\prime} \in X^{\prime}, F \in F B L(X)$ and $G \in B L(X)$. 


\section{Proof:}

\section{Proof(i)}

Let $(A,\|\cdot\|)$ be norm ideal on $X$, so by using Theorem 3.1 , we have $Q_{b}^{r}(A)=$ $B L(X)$ therefore $Q_{b e}^{r}(A) \subseteq B L(X)$, since $Q_{b e}^{r}(A)$ is a subalgebra of $Q_{b}^{r}(A)$ by [3, Theorem 1, p.117]. Now, let $T \in A$ and $F \in F B L(X)$, so $\|T\|_{r e}=\inf \left\{\left\|E_{T}^{I^{r}}\right\|:\right.$ I ideal of $A, T I \subseteq A, E_{T}^{I^{r}}$ bounded $\}$

Since $F B L(X)$ is an ideal of $B L(X)$ and every ideal of $B L(X)$ are contain $F B L(X)$ by [2, Theorem $4.3 .7(\mathrm{v}) \&(\mathrm{vi})$, p. 144] we get that

$$
\begin{aligned}
\|T\|_{r e} & =\left\|E_{T}^{F B L(X)^{r}}\right\| \\
& =\sup _{F \in F B L(X)^{r}}\left\{\left\|E_{T}^{F B L(X)^{r}}(F)\right\|,\|F\|=1\right\} \\
& =\sup _{F \in F B L(X)^{r}}\{\|F(T)\|,\|F\|=1\} \\
& \leq \sup _{F \in F B L(X)^{r}}\{\|F\|\|T\|,\|F\|=1\} \\
& =\|T\| \text { for all } T \in A .
\end{aligned}
$$

\section{Proof(ii)}

Let $(A,\|\cdot\|)$ be norm ideal on $X$. Then from [3, Theorem 1, p. 117] we get $Q_{b e}^{r}(A) \subseteq Q_{b}^{r}(A)$ and $\|S\|_{r} \leq\|S\|_{r e}$ for all $S \in Q_{b e}^{r}(A)$. Form Theorem 3.1 we have $\left(Q_{b}^{r}(A),\|\cdot\|_{r}\right)=\left(B L(X),\|\cdot\|_{\infty}\right)$ so $\|G\|_{\infty}=\|G\|_{r}$ for all $G \in Q_{b}^{r}(A)$. Thus $\|S\|_{\infty} \leq$ $\|S\|_{r e}$ for all $S \in Q_{b e}^{r}(A)$.

\section{Proof(iii)}

Let $x \in X$ and $y^{\prime} \in X^{\prime}$, then $x \otimes y^{\prime}$ is finite rank operator on complex Banach space $X$. So

$$
\begin{aligned}
& \|x\|\left\|y^{\prime}\right\|=\left\|x \otimes y^{\prime}\right\|_{\infty} \\
& \leq\left\|x \otimes y^{\prime}\right\|_{\text {re }} \text { from (ii) } \\
& \leq\left\|x \otimes y^{\prime}\right\| \quad \text { from (i) } \\
& =\|x\|\left\|y^{\prime}\right\|
\end{aligned}
$$

From properties of norm ideal, so we get that $\left\|x \otimes y^{\prime}\right\|_{r e}=\|x\|\left\|y^{\prime}\right\|$.

\section{Proof (iv)}

Let $S \in Q_{b e}^{r}(A)$ and $F \in F B L(X)$

$\|S F\|=\left\|R_{F}^{A}(S)\right\|$

$$
\leq\left\|E_{S}^{F B L(X)^{r}}\left(R_{F}^{A}\right)\right\|
$$

Since $F \in F B L(X)$, we have $R_{F}^{A} \in F B L(X)^{r}$.

$$
\leq\left\|E_{S}^{F B L(X)^{r}}\right\|\left\|R_{F}^{A}\right\|
$$

Since $S \in Q_{b e}^{r}(A)$, there exists an ideal $I$ of $A$ such that $S I \subseteq A$ and $E_{S}^{I^{r}}$ is bounded, $F B L(X)$ is an ideal of $B L(X)$ and every ideal of $B L(X)$ contain $F B L(X)$ by [2, Theorem 4.3.7(v), p. 144], so $F B L(X) \subseteq I$ and $E_{S}^{F B L(X)^{r}}$ is bounded. Now $\left\|R_{F}^{A}\right\|=\sup _{W \in A}\left\{\left\|R_{F}^{A}(W)\right\|,\|W\|=1\right\}$

$$
\begin{aligned}
& =\sup _{W \in A}\{\|W F\|,\|W\|=1\} \\
& =\sup _{W \in A}\left\{\left\|I d_{X} W F\right\|,\|W\|=1\right\} \\
& \leq \sup _{W \in A}\left\{\left\|I d_{X}\right\|_{\infty}\|W\|\|F\|_{\infty},\|W\|=1\right\}
\end{aligned}
$$

Since $F, I d_{X} \in B L(X)$ and from properties of norm ideal. 
$=\|F\|_{\infty}$

From (1) \&(2) we get

$\|S F\| \leq\left\|E_{S}^{F B L(X)^{r}}\right\|\|F\|_{\infty}$

$\leq\|S\|_{r e}\|F\|_{\infty}$ for all $S \in Q_{b e}^{r}(A)$ and $F \in F B L(X)$.

\section{Proof (v)}

Let $G \in B L(X)$ and $V \in F B L(X)^{r}$ for proving that $\left\|V R_{G}^{A}\right\| \leq\|V\|\|G\|_{\infty}$, first we will prove that $F B L(X)^{r} R_{G}^{A} \subseteq F B L(X)^{r}$. Let $T \in A$. Since $V \in F B L(X)^{r}$, then there exists $a_{i} \in A, x_{i} \in F B L(X)$ and $n \in \mathbb{N}$ such that $V=\sum_{i=1}^{n} M_{a_{i}, x_{i}}$. Now,

$$
\begin{aligned}
V R_{G}^{A}(T) & =\sum_{i=1}^{n} M_{a_{i}, x_{i}}\left(R_{G}^{A}(T)\right) \\
& =\sum_{i=1}^{n} M_{a_{i}, x_{i}}(T G) \\
& =\sum_{i=1}^{n} a_{i} T G x_{i} \\
& =\sum_{i=1}^{n} a_{i} T c_{i}, c_{i}=G x_{i} \\
& =\sum_{i=1}^{n} M_{a_{i}, c_{i}}(T)=V 1(T) \text { where } V 1=\sum_{i=1}^{n} M_{a_{i}, c_{i}}
\end{aligned}
$$

Since $c_{i} \in F B L(X)$ we have $V_{1} \in F B L(X)^{r}$, since $G \in B L(X)$ we get $R_{G}^{A} \in M(A)$, $F B L(X)^{r}$ is an ideal of $M(A)$ by [3, Lemma 1, p. 116], so $V R_{G}^{A} \in F B L(X)^{r}$ for all $V \in$ $F B L(X)^{r}$, then $F B L(X)^{r} R_{G}^{A} \subseteq F B L(X)^{r}$. Now to prove that $\left\|V R_{G}^{A}\right\| \leq\|V\|\|G\|_{\infty}$.

$$
\begin{aligned}
\left\|V R_{G}^{A}(T)\right\| & =\|V(T G)\| \\
& \leq\|V\|\|T G\|
\end{aligned}
$$

$T \in A$ and $G \in B L(X)$ then $T G \in A$ and $V$ is bounded.

$$
\begin{aligned}
& =\|V\|\left\|I d_{X} T G\right\| \\
& \leq\|V\|\left\|I d_{X}\right\|_{\infty}\|T\|\|G\|_{\infty}
\end{aligned}
$$

From properties of norm ideal

Now,

$$
\leq\|V\|\|T\|\|G\|_{\infty}
$$

$$
\begin{aligned}
\left\|V R_{G}^{A}\right\| & =\sup _{T \in A}\left\{\left\|V R_{G}^{A}(T)\right\|,\|T\|=1\right\} \\
& \leq \sup _{T \in A}\left\{\|V\|\|T\|\|G\|_{\infty},\|T\|=1\right\} \\
& =\|V\|\|G\|_{\infty}-------(3),
\end{aligned}
$$

for all $V \in F B L(X)^{r}$ and $G \in B L(X)$.

Let $S \in Q_{b e}^{r}(A)$, to prove that $\|S G\|_{r e} \leq\|S\|_{r e}\|G\|_{\infty}$

$$
\begin{aligned}
\left\|E_{S G}^{F B L(X)^{r}}(V)\right\| & =\|V(S G)\| \\
& =\left\|V R_{G}^{A}(S)\right\| \\
& =\left\|E_{S}^{F B L(X)^{r}}\left(V R_{G}^{A}\right)\right\|
\end{aligned}
$$

From above we get $V R_{G}^{A} \in F B L(X)^{r}$ 


$$
\leq\left\|E_{S}^{F B L(X)^{r}}\right\|\left\|V R_{G}^{A}\right\|
$$

Since $S \in Q_{b e}^{r}(A)$ we have $E_{S}^{F B L(X)^{r}}$ is bounded and from (3) we get

$$
\leq\left\|E_{S}^{F B L(X)^{r}}\right\|\|V\|\|G\|_{\infty}
$$

We get that $E_{S}^{F B L(X)^{r}}$ is bounded for all $G \in B L(X)$ and $S \in Q_{b e}^{r}(A)$, then $Q_{b e}^{r}(A)$ is right ideal of $B L(X)$.

$$
\begin{aligned}
\|S G\|_{r e} & =\left\|E_{S G}^{F B L(X)^{r}}\right\| \\
& =\sup _{V \in F B L(X)^{r}}\left\{\left\|E_{S G}^{F B L(X)^{r}}(V)\right\|,\|V\|=1\right\}, \text { from (4) we get } \\
& \leq \sup _{V \in F B L(X)^{r}}\left\{\left\|E_{S}^{F B L(X)^{r}}\right\|\|V\|\|G\|_{\infty},\|V\|=1\right\} \\
& =\left\|E_{S}^{F B L(X)^{r}}\right\|\|G\|_{\infty} \\
& =\|S\|_{r e}\|G\|_{\infty} \text { for all } S \in Q_{b e}^{r}(A) \text { and } G \in B L(X) .
\end{aligned}
$$

With similar proving for Proposition 3.2, we have the following proposition for the left algebra of quotients with bounded evaluation of norm ideal of complex Banach space.

\section{Proposition 3.3}

Let $(A,\|\cdot\|)$ be norm ideal on complex Banach space $X$ then $Q_{b e}^{l}(A)$ is a left ideal of $B L(X)$ and $\|\cdot\|_{l e}$ is norm have the following properties:

i. $\|T\|_{l e} \leq\|T\|$

ii. $\|S\|_{\infty} \leq\|S\|_{l e}$

iii. $\left\|x \otimes y^{\prime}\right\|_{l e}=\|x\|\left\|y^{\prime}\right\|$

iv. $\|S F\| \leq\|S\|_{l e}\|F\|_{\infty}$

v. $\|G S\|_{l e} \leq\|G\|_{\infty}\|S\|_{l e}$

For all $T \in A, S \in Q_{b e}^{l}(A), x \in X, y^{\prime} \in X^{\prime}, F \in F B L(X)$ and $G \in B L(X)$. 


\section{REFERENCES}

[1] Al-Neima, Mohammed Th. \& Mohammed, Amir A., (2020) "Proving the Equality of the Spaces $Q_{b}^{r}(A), Q_{b}^{l}(A)$ and $B L(X)$ Where $X$ is a Complex Banach Space", Iraqi journal for science, Vol. 61, No. 1, p. 127-131.

[2] Beidar, K. I., Martindale 3rd, W. S., and Mikhalev, A. V. (1995) "Rings with generalized identities" CRC Press.

[3] Cabrera, M., \& Mohammed, A. A. (2003) "Algebras of quotients with bounded evaluation of a normed semiprime algebra" Studia Mathematica, Vol. 154, No. 2, pp. 113-135.

[4] García, M. C., \& Palacios, Á. R. (1990) "Nonassociative ultraprime normed algebras" Extracta mathematicae, Vol. 5, No. 3, pp. 127-129.

[5] García, M. C., \& Palacios, A. R. (1992) "Nonassociative ultraprime normed algebras" The Quarterly Journal of Mathematics, Vol. 43, No. 1, pp. 1-7.

[6] García, M. C., \& Palacios, Á. R. (2014) "Non-Associative Normed Algebras: Volume 1, The Vidav-Palmer and Gelfand-Naimark Theorems" (Vol. 154) Cambridge University Press.

[7] García, M. C., \& Palacios, Á. R. (2018) "Non-associative normed algebras. Volume 2: Representation Theory and the Zel'manov Approach" Encyclopedia Math. Appl., Vol.(167), Cambridge University Press.

[8] Mathieu, M. (1986) "Applications of ultraprime Banach algebras in the theory of elementary operators" Ph.D. Thesis,University of Tübingen, Germany.

[9] Mathieu, M. (1989) "Rings of quotients of ultraprime Banach algebras: with applications to elementary operators" In Mini conference on Operators in Analysis. Centre for Mathematics and its Applications, Mathematical Sciences Institute. The Australian National University. pp. 297-317. 